\title{
Notch pathway activation promotes the differentiation of beagle dog periodontal ligament stem cells to Schwann cells
}

\author{
Xiaojie Li ${ }^{1, A-F}$, Dapeng Liao ${ }^{2, A-F}$, Gang Sun ${ }^{2, B, C, E, F}$, HanWen Chu ${ }^{2, B, C, E, F}$ \\ ${ }^{1}$ Department of Dentistry, Sir Run Run Shaw Hospital, Zhejiang University School of Medicine, China \\ ${ }^{2}$ Department of Dentistry, The Second Affiliated Hospital, Zhejiang University School of Medicine, China \\ A - research concept and design; $\mathrm{B}$ - collection and/or assembly of data; $\mathrm{C}$ - data analysis and interpretation; \\ $D$ - writing the article; $E$ - critical revision of the article; $F$ - final approval of the article
}

\section{Address for correspondence}

Xiaojie Li

E-mail:3312026@zju.edu.cn

\section{Funding sources}

The Project was supported by National Natural Science Foundation of China under grant $N 0.81702222$ and Zhejiang Provincial Natural Science Foundation of China under grant N No. LQ17H170001.

Conflict of interest

None declared

Received on July 8,2020

Reviewed on July 15,2020

Accepted on December 6, 2020

Published online on Juni 11, 2021

\begin{abstract}
Background. Periodontal ligament stem cells (PDLSCs) have demonstrated the potential for differentiation into many cell types, though the molecular mechanism of their neural differentiation in particular remains largely unknown.
\end{abstract}

Objectives. The Notch signaling pathway plays a key role in regulating cell differentiation and development. In this article, we explore its potential role in the differentiation of PDLSCs to Schwann cells (SCS).

Materials and methods. The PDLSCs were either transfected with viral vectors carrying genetic material for Notch Delta ligands, thereby induced their overexpression, or treated with DAPT (a Notch-pathway-specific inhibitor) to inhibity-secretase. The potential effects of Notch signaling on myelination and SCs differentiation were then investigated using western blotting, immunostaining and reverse transcriptase polymerase chain reaction (RT-PCR) to detect the expression of SC-specific marker genes.

Results. Specifically inhibiting Notch signaling with DAPT decreased the expression of SC-specific marker genes GFAP, S100 and P75, as well as of SC-myelin-related genes PMP22, MBP, connexin, and PO in cells undergoing induced differentiation from PDLSCs. Conversely, activating Notch signaling through overexpression of Delta ligands enhanced the expression of SC-specific marker genes as well as myelin-related genes in cells undergoing induced differentiation from PDLSCS. This promotion was reversed by DAPT.

Conclusions. The Notch signaling pathway positively regulated the process of PDLSC differentiation into SCs, and the activation of this signaling was important in maintaining the differentiation of PDLSCS to SCS, and then SC myelination. These results may improve the method of obtaining pure SCs from PDLSCS for transplantation application.

Key words: differentiation, Schwann cells, periodontal ligament stem cells, Notch signaling pathway

Cite as

Li X, Liao D, Sun G, Chu HW. Notch pathway activation promotes the differentiation of beagle dog periodontal ligament stem cells to Schwann cells. Adv Clin Exp Med. 2021;30(7):721-726. doi:10.17219/acem/131219

DOI

10.17219/acem/131219

Copyright

Copyright by Author(s)

This is an article distributed under the terms of the

Creative Commons Attribution 3.0 Unported (CC BY 3.0)

(https://creativecommons.org/licenses/by/3.0/) 


\section{Background}

The Schwann cells (SCs) are a glial nerve cell type that form an important part of the Ruffini body of periodontal nerve endings, and play an important role in growth, development and regeneration of peripheral nerves. ${ }^{1,2}$ Schwann cells are essential in increasing the density of peripheral nerve endings, and in the improvement of osseoperception. They respond rapidly to nerve injury, and promote axon regrowth and nerve regeneration. Schwann cells also produce various growth factors that are involved in phagocytosis and the clearance of myelin fragments. All these characteristics have made SCs the first and the most widely used support cells to be used for peripheral nerve regeneration. ${ }^{3-5}$

In our previous study, we found that periodontal ligament stem cells (PDLSCs) isolated from beagle dogs effectively differentiated into SCs with exposure to a combination of dimethyl sulfoxide (DMSO), basic fibroblast growth factor (bFGF), brain-derived neurotrophic factor (BDNF), nerve growth factor (NGF) and forskolin. ${ }^{6,7} \mathrm{Mul}-$ tiple signaling pathways, transcription factors, and neurotrophic factors had been implicated in their differentiation. ${ }^{3,8}$ However, the mechanisms underlying the process of PDLSC differentiation to SCs are poorly characterized.

We have previously demonstrated that the Erk1/2 signaling pathway is involved in the differentiation of PDLSCs to SCs. ${ }^{9}$ Although inhibition of the Erk $1 / 2$ pathway is shown to prevent the differentiation of PDLSCs to SCs, we still find evidence of a number of SCs in culture. This suggests that other signaling pathways are also involved in the process.

In this study, we investigate whether the Notch signaling pathway is involved in the differentiation of PDLSCs to SCs. The Notch signaling pathway is a highly conserved system that regulates cell differentiation and development in many multicellular organisms..$^{10,11}$ Moreover, many reports indicate that Notch signaling is involved in different stages of SCs development, including acting on neural crest stem cells and mediating the generation of immature SCs. ${ }^{12,13}$ The structure of Notch family members is highly conserved, and the regulatory mechanism is very complex. Notch signaling in vertebrates consists of Notch, Notch ligands (Delta and Jagged proteins) and CSL DNA binding proteins. Following Notch receptor binding to Delta or Jagged ligands, Notch is cleaved by the $\gamma$-secretase activity of presenilin-1 (PS1) to release the cytoplasmic domain of the Notch receptor - the Notch intracellular domain (NIC) - which translocates to the nucleus and binds to the transcription factor CSL, leading to the transcriptional activation of downstream target genes. ${ }^{14}$

\section{Objectives}

In this study, we provide evidence that Notch signaling represents a key regulatory pathway in regulating PDLSC differentiation. We used the $\gamma$-secretase inhibitor DAPT to specifically inhibit the Notch signaling pathway, and overexpressed the Delta ligand to activate it. The effect of the Notch signaling pathway on the differentiation of PDLSCs to SCs was examined.

\section{Materials and methods}

All animals were purchased from Sichuan University and the experimental protocol was approved by the Ethical Guideline Committee of Animal Care, West China College of Medical Sciences, Sichuan University, Chengdu, China.

\section{Isolation and culture of PDLSCs}

The PDLSCs were isolated from the periodontal ligaments of beagle dogs using the single-colony selection method as described previously. ${ }^{15}$ Two healthy beagle dogs, 12-month-old, weighing about $10 \mathrm{~kg}$, were selected. The premolars were extracted, and the periodontal ligaments were carefully scraped off. The PDLSCs were dissociated by digesting the periodontal ligaments with TrypsinEDTA (0.25\%) (Catalog No. 25200056; Gibco, Waltham, USA) and cultured following gradient centrifugation. Next, PDLSCs were identified by a combination of optical microscopy and flow cytometry, as described previously. ${ }^{16}$

\section{Differentiation of PDLSCs to SCs}

We induced the differentiation of PDLSCs to SCs as described previously. ${ }^{6}$ The PDLSCs were then treated with $2 \%$ DMSO for $5 \mathrm{~h}$, before culturing in differentiation medium, which contained $10 \mathrm{ng} / \mathrm{mL}$ bFGF, $10 \mathrm{ng} / \mathrm{mL}$ NGF, $10 \mathrm{ng} / \mathrm{mL}$ BDNF, $14 \mathrm{mM}$ forskolin, and 20\% (v/v) fetal bovine serum (FBS) in minimal essential medium (MEM) alpha modification, with L-glutamine, with ribo- and deoxyribonucleosides (Sigma-Aldrich, St. Louis, USA).

\section{Co-culture of PDLSCs and DRG cells}

For co-culture assays, cells were grown in six-cell culture inserts following the manufacturer's instructions (BD Biosciences, Franklin Lakes, USA). A population of $1 \times 10^{4}$ dorsal root ganglion (DRG) cells was diluted in $1 \mathrm{~mL}$ medium and loaded into the upper chamber of a transwell cell insert (Lot 3450; Corning Inc., Corning, USA), while a population of $2 \times 10^{4}$ PDLSCs in $2 \mathrm{~mL}$ medium was added to the lower chambers. The transwell cell inserts allowed PDLSCs and DRG cells to share the same medium without direct contact.

\section{Western blotting}

S100, GFAP, P75, MYT1L, SNW1, ASPN, and GAPDH antibodies were purchased from Cell Signaling Technology 
(Danvers, USA) and diluted at 1:100 in Tris-buffered saline with Tween (TBST). Total protein was extracted using RIPA lysis buffer, and protein concentration was determined using a bicinchoninic acid (BCA) assay kit (M\&E Gene Technology, Beijing, China). Western blotting was performed as described previously. ${ }^{9}$

\section{Immunofluorescence}

Protein zero (P0) and myelin basic protein (MBP) antibodies were purchased from Abcam (Cambridge, UK). DAPT and fluorescein isothiocyanate-conjugated secondary antibody were purchased from BD Biosciences. After 2 weeks of induction, cells were immunoassayed as described previously. ${ }^{9}$ Immunofluorescence was visualized under an Olympus IX 71 microscope (Olympus Corp., Tokyo, Japan).

\section{Quantitative real-time PCR}

Total RNA was extracted using Trizol reagent according to the manufacturer's guidelines. Reverse transcription of mRNA was performed using the PrimeScript RT kit (TaKaRa, Kyoto, Japan). The quantitative real-time polymerase chain reaction (qRT-PCR) was performed on an ABI 7500 (Applied Biosystems, Foster City, USA) using a SYBR Premix Ex Taq Kit (TaKaRa) according to the manufacturer's guidelines. GAPDH was used as an internal control.

\section{Statistical analyses}

Each experiment was performed 3 times. One-way analysis of variance (ANOVA) and the Student's t-test were used to determine statistical differences between groups. All data are expressed as mean \pm standard deviation (SD). A value of $\mathrm{p}<0.05$ was considered significant.

\section{Results}

\section{Characterization of PDLSCs}

The PDLSCs appeared mainly polygonal with a typical spindle-shaped, fibroblast-like morphology (Fig. 1A). Flow cytometry was performed to detect stem cell surface markers. These cells showed relatively high expression levels of the surface markers STRO-1 and CD146 (Fig. 1B), indicating that they indeed had the characteristics of stem cells. The concentration of PDLSCs in the sample was very high.

\section{Activation of the Notch signaling pathway promotes the differentiation of PDLSCs into SCs}

Firstly, we characterized Notch signaling in the differentiation of PDLSCs into SCs. The PDLSCs were cultured

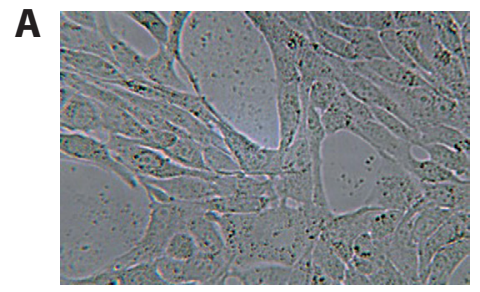

B
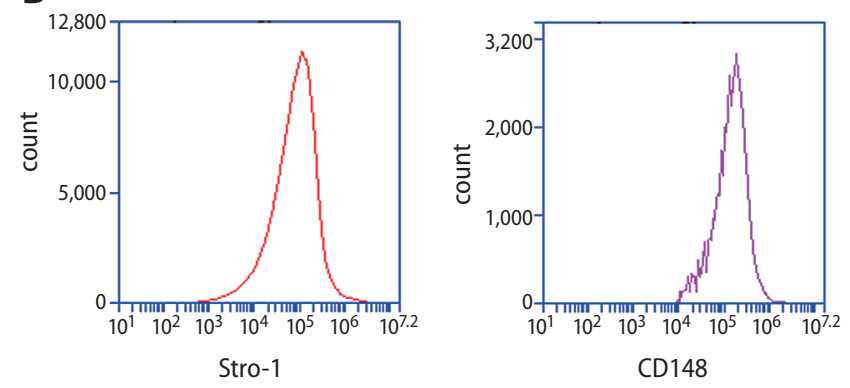

Fig. 1. Identification of PDLSCs. A. Inverted microscopy revealing the cell morphology of PDLSCs; B. Detection of PDLSC markers using flow cytometry

in differentiation medium containing DMSO, bFGF, BDNF, NGF, and forskolin to induce SC phenotypes. Differentiation into SCs was confirmed by the high expression of SC protein markers, including S100, GFAP and P75, as detected using western blot. We also found that differentiated PDLSCs exhibited upregulation of key proteins in the Notch 1 signaling pathway compared with undifferentiated PDLSCs (Fig. 2), such as the myelin transcription factor 1-like protein (MYT1L), a coactivator for Notch transcriptional activation, SNW domain-containing protein 1 (SNW1) and ASPN. These results suggested that the Notch pathway was activated during the differentiation of PDLSCs to SCs.

To further evaluate whether the Notch pathway was involved in the differentiation of PDLSCs into $\mathrm{SCs}$, we investigated the effect of activation or inhibition of Notch signaling, using viruses overexpressing the Delta Notch ligand or an inhibitor of the Notch pathway, respectively. We found that activation of Notch signaling through the overexpression of the Delta ligand (which was confirmed by the upregulation of the MYT1L protein) increased the expression of SC-specific markers, including S100, GFAP and P75, compared to the control group. Further, the addition of DAPT to the differentiation medium decreased the expression of SC-specific markers compared with the control group (Fig. 3). These data suggest that activation of Notch signaling promotes the differentiation of PDLSCs into SCs, while inhibition of Notch signaling hinders this process. Taking all of these evidences into account, it appears that the Notch signaling pathway is involved in the differentiation of PDLSCs into SCs. 


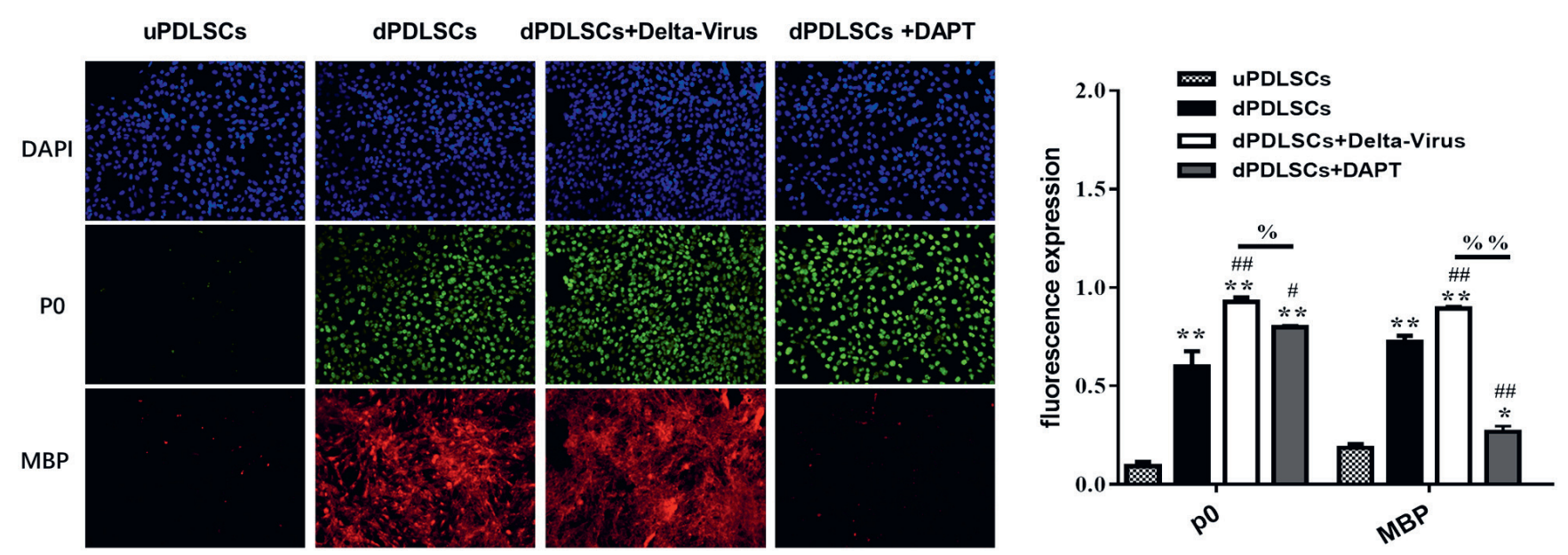

Fig. 2. Notch signaling pathway is involved in myelin formation during the differentiation of PDLSCs to SCs. Co-culture of PDLSCS and DRGs was conducted as described in the legend of Fig. 3. The expression of SC myelin-formation-related proteins PO and MBP was analyzed with immunofluorescent staining. Blue spots represent nuclei stained with DAPI

${ }^{*} p<0.05,{ }^{* *} p<0.01$ compared to uPDLSC group; ${ }^{*} p<0.05,{ }^{\# \#} p<0.01$ compared to corresponding dPDLSC group; ${ }^{\%} p<0.05,{ }^{\%} \% p<0.01$ compared to dPDLSC+Delta-Virus group.

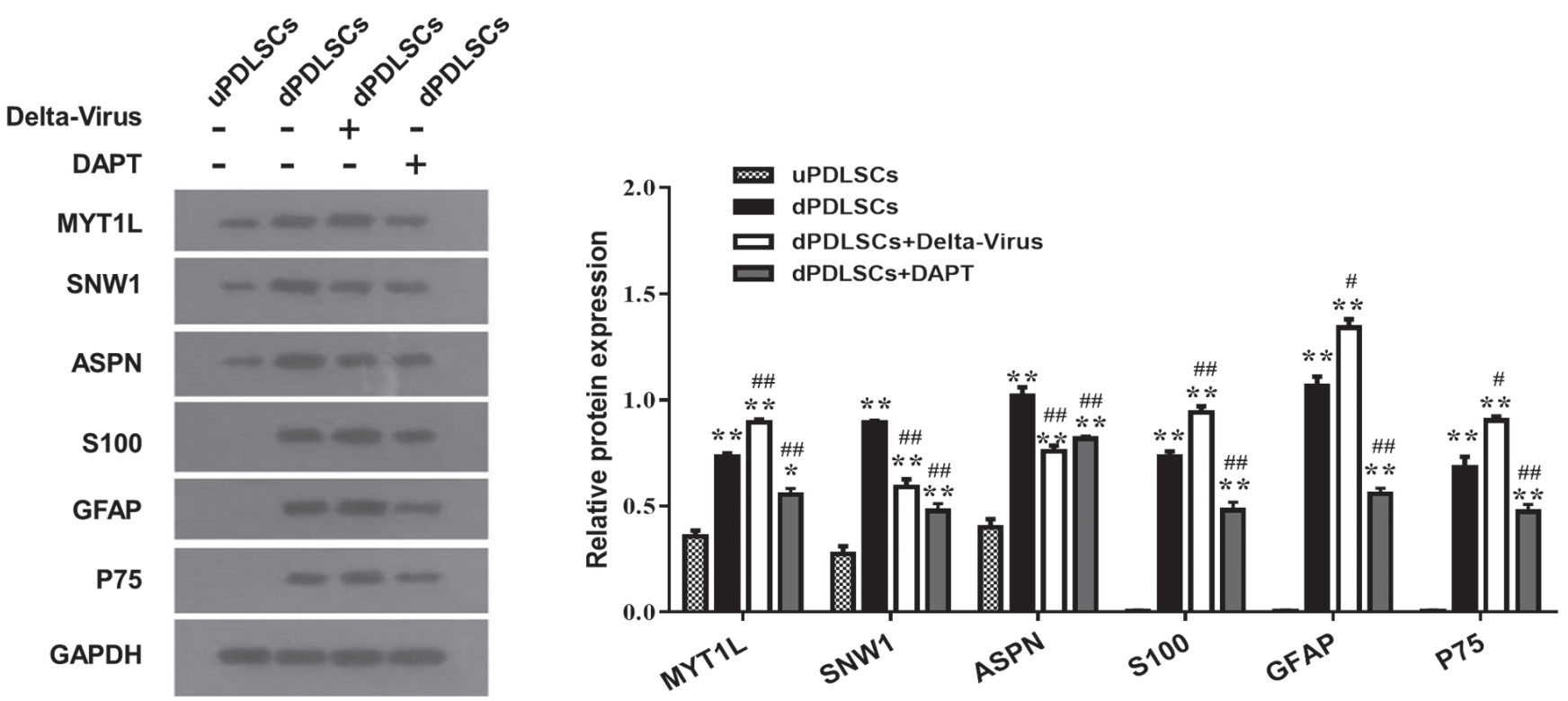

Fig. 3. Notch signaling pathway is involved in differentiation of PDLSCs to SCs. PDLSCs were induced to differentiate into SCs. In one group, PDLSCs were transfected by viral vector expressing the Delta ligand. In the other group, DAPT was added to the medium to specifically inhibit the Notch signaling pathway. Protein was extracted from: undifferentiated PDLSCs (uPDLSCs); differentiated PDLSCs (dPDLSCs); differentiated PDLSCs transfected with the Delta viral vector; or differentiated PDLSCs treated with DAPT. The expression of Notch-pathway-related proteins MYT1L, SNW1 and ASPN, as well as SC-specific proteins S100, GFAP and P75, was analyzed with western blotting. Relative protein expressions were normalized to levels of GAPDH

${ }^{*} p<0.05,{ }^{* *} p<0.01$ compared to UPDLSC group; ${ }^{*} p<0.05,{ }^{\# \#} p<0.01$ compared to corresponding dPDLSC group.

\section{Activation of Notch signaling promotes myelin formation during differentiation of PDLSCs into SCs}

We co-cultured PDLSCs with dorsal DRG cells to evaluate the effect of Notch signaling on myelin formation during the differentiation of PDLSCs into SCs. Immunostaining was performed in order to assess myelin formation. We found that co-culture with DRG under induced differentiation conditions significantly promoted the expression of P0 and MBP in the cytoplasm of differentiated PDLSCs, confirming that PDLSCs were induced to differentiate into SCs (Fig. 3). We further evaluated whether changes in Notch signaling would affect the expression of these genes in differentiated PDLSCs that had been co-cultured with DRG. We found that the overexpression of Delta in PDLSCs promoted the expression of P0 and MBP, and that differentiated cells treated with DAPT showed decreased P0 and MBP expression (Fig. 3). The RT-PCR results showed that the expression of myelin-related genes 


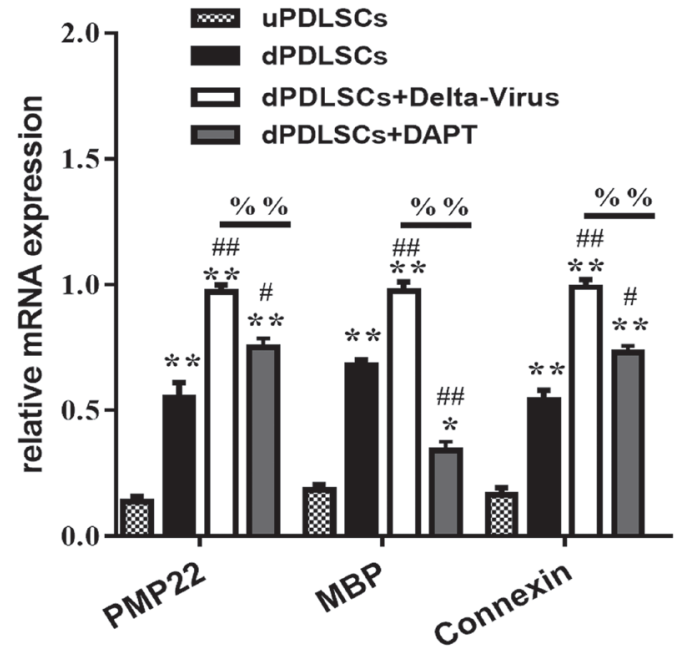

Fig. 4. Myelin-related genes in SCS were changed during the differentiation of PDLSCS to SCs. The expression of SC myelinformation-related-genes PMP22, MBP and connexin was analyzed using RT-PCR

${ }^{*} \mathrm{p}<0.05,{ }^{* *} \mathrm{p}<0.01$ compared to uPDLSC group; $\mathrm{*}<0.05$;

$\# \mathrm{p}<0.01$ compared to the corresponding $\mathrm{dPDLSC}$ group;

${ }^{\%} \mathrm{p}<0.05,{ }^{\% \%} \mathrm{p}<0.01$ compared to dPDLSC+Delta-Virus group.

(including PMP22, MBP and connexin) were upregulated in the Delta overexpression group compared with control group, but downregulated in DAPT-treated cells (Fig. 4). These results suggest that activation of Notch signaling promotes myelin formation during the differentiation of PDLSCs into SCs, while the inhibition of this signaling impairs myelin formation under the differentiation condition.

\section{Discussion}

In recent years, it has been confirmed that the Notch signaling pathway plays an important role in regulating the differentiation of adult stem cells into SCs. ${ }^{11} \mathrm{We}$ previously demonstrated that PDLSCs isolated from beagle dogs could differentiate into SCs, and that this differentiation, as well as SC myelination, was interrupted by specific inhibition of the Erk1/2 signaling pathway. ${ }^{6,9}$

In this study, we demonstrated that Notch signaling was important in promoting the differentiation of PDLSCs into SCs. First, we found that protein levels of MYT1L, SNW1 and ASPN were upregulated when PDLSCs were cultured in induction media. MYT1L, SNW1 and ASPN act as coactivators for Notch transcriptional activation, ${ }^{16}$ indicating that the Notch pathway was activated in the differentiation process of PDLSCs into SCs. Next, we found that inhibition of the Notch pathway in differentiated cells by treating with a $\gamma$-secretase inhibitor decreased the expression of SC-specific markers S100, GFAP and P75. Meanwhile, the activation of the Notch pathway through the overexpression of Delta increased the expression of these markers.
Notch has complex and extensive regulatory functions in SCs. In primary SCs isolated from neonatal rats, Notch activation promotes the generation of SCs, and negatively regulates SC myelination. ${ }^{12,13}$ Moreover, in contrast to primary SCs, Notch signaling has no effect on the neurotrophic activity and myelination capability of adiposederived stem cells (ASCs) undergoing differentiation into $\mathrm{SCs},{ }^{17}$ indicating that the effect of Notch on gliogenesis is not universal. On the contrary, we showed that specific inhibition of the Notch pathway downregulated the expression of SC myelination-related genes (including $P 0, M B P$, $P M P 22$, and connexin), whereas activation of the Notch pathway increased the expression of these genes in DRG neurons. Our findings raise the possibility that the activation of Notch signaling in PDLSCs may initiate myelination by upregulating SC myelination-related genes.

\section{Limitations}

There are some limitations of this study. First, differentiation into SCs was only investigated in PDLSCs isolated from the periodontal ligaments of beagle dogs. The current findings need to be validated in primary human cells and animal models. Second, the Notch signaling pathway is comprised of a complex network. Many other signaling molecules related to PDLSC differentiation and Notch signaling remain to be investigated in future researches.

\section{Conclusions}

The genes associated with the Notch pathway were upregulated in differentiated PDLSCs, and the activation of the Notch pathway through the overexpression of Delta ligands promoted the differentiation of PDLSCs into SCs, as well as SC myelination. In contrast, the inhibition of the Notch pathway by DAPT treatment prevented the differentiation of PDLSCs into SCs, and also SC myelination. Therefore, the Notch signaling pathway appears to positively regulate the process of PDLSC differentiation into SCs, and the activation of this signaling is important in maintaining that differentiation, as well as SC myelination. These results may improve methods of obtaining pure SCs from PDLSCs for transplantation use.

\section{ORCID iDs}

Xiaojie Li (D) https://orcid.org/ 0000-0002-6363-6255 Dapeng Liao (D) https://orcid.org/ 0000-0001-6751-7838 Gang Sun (D) https://orcid.org/ 0000-0002-5353-3463 HanWen Chu (D) https://orcid.org/ 0000-0001-8289-0593

\section{References}

1. Mirsky R, Jessen KR. Schwann cell development, differentiation and myelination. Curr Opin Neurobiol. 1996;6(1):89-96. doi:10.1016/s09594388(96)80013-4

2. Frostick SP, Yin Q, Kemp GJ. Schwann cells, neurotrophic factors, and peripheral nerve regeneration. Microsurgery. 1998;18(7):397-405. doi:10.1002/(sici)1098-2752(1998)18:7<397::aid-micr2>3.0.co;2-f 
3. Jessen KR, Mirsky R. The origin and development of glial cells in peripheral nerves. Nat Rev Neurosci. 2005;6(9):671-682. doi:10.1038/nrn1746

4. Hammarberg $\mathrm{H}$, Risling M, Hokfelt T, Cullheim S, Piehl F. Expression of insulin-like growth factors and corresponding binding proteins (IGFBP 1-6) in rat spinal cord and peripheral nerve after axonal injuries. J Comp Neurol. 1998;400(1):57-72. PMID:9762866

5. Mosahebi A, Woodward B, Wiberg M, Martin R, Terenghi G. Retroviral labeling of Schwann cells: In vitro characterization and in vivo transplantation to improve peripheral nerve regeneration. Glia. 2001; 34(1):8-17. doi:10.1002/glia.1035

6. Li X, Gong P, Liao D. In vitro neural/glial differentiation potential of periodontal ligament stem cells. Arch Med Sci. 2010;6(5):678-685. doi:10.5114/aoms.2010.17080

7. Li X, Liao D, Gong P, Dong Y, Sun G. Biological behavior of neurally differentiated periodontal ligament stem cells on different titanium implant surfaces. J Biomed Mater Res A. 2014;102(8):2805-2812. doi:10.1002/jbm.a.34953

8. Ijuin K, Nakanishi K, Ito K. Different downstream pathways for Notch signaling are required for gliogenic and chondrogenic specification of mouse mesencephalic neural crest cells. Mech Dev. 2008;125(5-6): 462-474. doi:10.1016/j.mod.2008.01.008

9. Dapeng L, Xiaojie L, Ping G, Yan D, Gang S. Erk1/2 signalling is involved in the differentiation of periodontal ligament stem cells to Schwann cells in dog. Arch Oral Biol. 2014;59(5):487-491. doi:10.1016/j.archoralbio. 2014.02.010
10. Joseph NM, Mukouyama YS, Mosher JT, et al. Neural crest stem cells undergo multilineage differentiation in developing peripheral nerves to generate endoneurial fibroblasts in addition to Schwann cells. Development. 2004;131(22):5599-5612. doi:10.1242/dev.01429

11. Shi Y, Shao Q, Li Z, et al. Myt1L promotes differentiation of oligodendrocyte precursor cells and is necessary for remyelination after lysolecithin-induced demyelination. Neurosci Bull. 2018;34(2):247-260. doi:10.1007/s12264-018-0207-9

12. Woodhoo A, Alonso MB, Droggiti A, et al. Notch controls embryonic Schwann cell differentiation, postnatal myelination and adult plasticity. Nat Neurosci. 2009;12(7):839-847. doi:10.1038/nn.2323

13. Wu LM, Wang J, Conidi A, et al. Zeb2 recruits HDAC-NuRD to inhibit Notch and controls Schwann cell differentiation and remyelination. Nat Neurosci. 2016;19(8):1060-1072. doi:10.1038/nn.4322

14. Fiuza UM, Arias AM. Cell and molecular biology of Notch. J Endocrinol. 2007;194(3):459-474. doi:10.1677/JOE-07-0242

15. Seo BM, Miura M, Gronthos S, et al. Investigation of multipotent postnatal stem cells from human periodontal ligament. Lancet. 2004; 364(9429):149-155. doi:10.1016/S0140-6736(04)16627-0

16. Vasquez-Del Carpio R, Kaplan FM, Weaver KL, et al. Assembly of a Notch transcriptional activation complex requires multimerization. Mol Cell Biol. 2011;31(7):1396-1408. doi:10.1128/MCB.00360-10

17. Kingham PJ, Mantovani $C$, Terenghi $G$. Notch independent signalling mediates Schwann cell-like differentiation of adipose derived stem cells. Neurosci Lett. 2009;467(2):164-168. doi:10.1016/j.neulet. 2009.10.030 